\title{
Low expression of Mig-6 is associated with poor survival outcome in NSCLC and inhibits cell apoptosis via ERK-mediated upregulation of Bcl-2
}

\author{
ZIXUAN LI ${ }^{1,3}$, LIANYUE QU $^{2}$, HONGSHAN ZHONG ${ }^{3}, \mathrm{KE} \mathrm{XU}^{3}, \mathrm{XUESHAN} \mathrm{QIU}^{1}$ and ENHUA WANG ${ }^{1}$
}

\begin{abstract}
${ }^{1}$ Department of Pathology, The First Affiliated Hospital of China Medical University and College of Basic Medical Sciences, China Medical University, Shenyang; Departments of ${ }^{2}$ Pharmacy and ${ }^{3}$ Radiology and Key Laboratory of Diagnostic Imaging and Interventional Radiology, The First Affiliated Hospital of China Medical University, Shenyang, Liaoning 110001, P.R. China
\end{abstract}

Received December 27, 2013; Accepted February 7, 2014

DOI: 10.3892/or.2014.3050

\begin{abstract}
Mitogen-inducible gene-6 (Mig-6), an immediate early response gene, is a specific negative regulator of epidermal growth factor receptor (EGFR). Ablation of Mig-6 has been shown to induce tumor formation in various tissues, supporting the tumor suppressor function of Mig-6. However, little is known about the role of Mig-6 in non-small cell lung cancer (NSCLC) apoptosis, nor has the contribution of upregulated Mig- 6 on biological behaviors of A549 and H157 cells previously been reported. The aim of the present study was to investigate the effects of exogenously transfected Mig-6 on proliferation, invasion and apoptosis of A549 and H157 cells and to identify novel underlying mechanisms of Mig-6induced apoptosis. We used immunohistochemical staining to examine the expression of Mig-6 protein in NSCLC tissues. For evaluation of the prognostic value of Mig- 6 expression to each clinicopathologic factor, Kaplan-Meier method and Cox's proportional hazards model were employed. Mig- 6 low expression was correlated with a poor prognosis in patients with lung cancer. Patients with high expression of Mig-6 had a statistically significantly longer survival than those with low expression of Mig-6. Cox's regression analysis indicated that loss of Mig-6 expression was an independent, unfavorable prognostic factors. We utilized siRNA-targeting Mig-6 and Mig- 6 overexpression plasmid to determine the effect of Mig- 6 on lung cancer cells. Flow cytometry studies revealed Mig-6 overexpression promoted apoptosis in NSCLC cell lines. siRNA-mediated Mig- 6 knockdown inhibited apoptosis of cancer cells, but this anti-apoptotic effect was abolished by inhibition of ERK. Upregulation of Mig- 6 decreased the proliferation and invasive potential of transfected cells.
\end{abstract}

Correspondence to: Dr Xueshan Qiu, Department of Pathology, The First Affiliated Hospital of China Medical University and College of Basic Medical Sciences, China Medical University, Shenyang, Liaoning 110001, P.R. China

E-mail: qiuxues@hotmail.com

Key words: mitogen-inducible gene-6, NSCLC, apoptosis, Bcl-2, p-ERK
Moreover, upregulation of Mig-6 inhibited proliferation and invasion of A549 and H157 cells. Collectively, our results showed that Mig- 6 is a potential biomarker for evaluation of tumor prognosis of lung cancer. Mig-6 promotes apoptosis in lung cancer cells via the ERK pathway.

\section{Introduction}

Mitogen-inducible gene-6 (Mig-6) is an immediate early response gene that can be induced by a variety of external stimuli, including growth factors, hypoxia and stress (1). The Mig-6 locus (chromosome 1p36.12-33) falls within the region 1p36.1-3 and exhibits a high frequency of allelic loss in various human cancer types (2-4). Mig-6 downregulation has been reported in various cancer types, and accumulating evidence suggests that Mig-6 is a tumor-suppressor gene in both mice and humans (5-9). A study by Amatschek et al (13) found that Mig- 6 is downregulated in breast cancer patients with short survival time. That study indicated that Mig- 6 may have a potential prognostic value in human cancer. Mig-6 binds to epidermal growth factor receptor (EGFR) family tyrosine kinases via its EGFR-binding domain thus leading to inhibition of EGFR autophosphorylation and reduced MAPK activity (10-13). Many experiments confirmed that Mig-6 exhibits a measurable effect on tumor cell proliferation and invasion, but its role in apoptosis remains unclear.

Our previously reported study demonstrated that Mig-6 is downregulated in non-small cell lung cancer (NSCLC) and knockdown of Mig- 6 expression in H1299 and BE1 cells promotes EGF-induced lung cancer cell proliferation and migration (14). However, little is known about the role of Mig-6 in NSCLC apoptosis, nor has the contribution of upregulated Mig- 6 on biological behaviors of A549 and H157 cells previously been reported.

In the present study, statistical analysis was performed on the potential correlation between Mig- 6 expression and poor survival outcome after lung cancer surgery. The increase of the Mig-6 expression affecting proliferation, invasion of A549 and $\mathrm{H} 157$ cells were examined. We also investigated the effects and regulatory mechanisms underlying the impact of Mig-6 on apoptosis in human NSCLC cells. 


\section{Materials and methods}

Patients and specimens. The present study was conducted with the approval of the Ethics Committee of China Medical University. Primary tumor specimens were obtained from the First Affiliated Hospital of China Medical University during the period of 2008 to 2013. Through the surgery consent form, patients were informed that the resected specimens were kept by our hospital and may be used for scientific research, and that their privacy would be maintained. Follow-up information was obtained by reviewing patient medical records. None of the patients received radiotherapy or chemotherapy before surgical resection, and all patients were treated with routine chemotherapy after the operation. The histological diagnosis and grade of differentiation of the tumors were defined by evaluation of the hematoxylin and eosin-stained tissue sections, according to the World Health Organization guidelines of classification. All 150 specimens were re-evaluated with respect to their histological subtypes, differentiation status and tumor stages. For NSCLC samples, squamous-cell carcinoma (SCC) and adenocarcinoma were identified in 69 and 81 of the 150 cases, respectively. Lymph node metastases were observed in 60 of the 150 patients. The p-TNM staging system of the International Union Against Cancer (7th edition) was used to classify specimens.

Cell culture and transfection. A549 and H1299 were obtained from the American Type Culture Collection (Manassas, VA, USA), and H157 was purchased from the Cell Bank, Chinese Academy of Sciences (Shanghai, China). The BE1 were gifts from Dr Jie Zheng (College of Medicine, Beijing University, China). The cells were cultured in RPMI-1640 containing $10 \%$ fetal calf serum (both from Invitrogen), $100 \mathrm{IU} / \mathrm{ml}$ penicillin and $100 \mu \mathrm{g} / \mathrm{ml}$ streptomycin (both from Sigma). The cells were seeded in 6 -well plates $24 \mathrm{~h}$ prior to the experiment. Transfection with siRNA for Mig-6 or non-targeting siRNA (GenePharma, Shanghai, China), was conducted using DharmaFECT 1 (5 $\mu \mathrm{l} /$ well; Thermo Fisher Scientific, Rockford, IL, USA) according to the manufacturer's recommendations. The sequences of siRNAs were: sense, 5'-GGAUAUCCAACUGUUGUAUTT-3' and antisense, 5'-AU ACAACAGUUGGAUAUCCTT-3' (6). For control scrambled siRNA: sense, 5'-UUCUCCGAACGUGUCACGUTT-3' and antisense, 5'-ACGUGACACGUUCGGAGAATT-3'. Cells were transfected with control vector (pcDNA3) or Mig-6 overexpression vector (pcDNA3-Mig-6) (both from Takara Biotechnology, Dalian, China) using Attractene transfection reagent (Qiagen, Valencia, CA, USA) according to the manufacturer's protocol. The mRNA and protein levels were assessed $48 \mathrm{~h}$ following transfection.

Immunohistochemistry. Surgically excised tumor specimens were fixed with $10 \%$ neutral formalin, embedded in paraffin and $4-\mu \mathrm{m}$ thick sections were prepared. Immunostaining was performed using the avidin-biotin-peroxidase complex method (Ultra Sensitive TM, Maixin, Fuzhou, China). The sections were deparaffinized in xylene, rehydrated in graded alcohol series and boiled in $0.01 \mathrm{M}$ citrate buffer ( $\mathrm{pH}$ 6.0) for $2 \mathrm{~min}$ in an autoclave. Endogenous peroxidase activity was blocked using hydrogen peroxide $(0.3 \%)$, which was followed by incubation with normal goat serum to reduce non-specific binding. Tissue sections were incubated with Mig- 6 rabbit polyclonal antibody (1:100 dilution; Sigma). Rabbit immunoglobulin (at the same concentration as the antigen specific antibody; Maixin) was used as a negative control. Staining for all primary antibodies was performed at room temperature for $2 \mathrm{~h}$. HRP-polymer conjugated anti-mouse/rabbit IgG (ready-to-use; Maixin) was used as the secondary antibody. After washing, the sections were incubated with horseradish peroxidase-conjugated streptavidin-biotin, followed by 3,3'-diaminobenzidine tetrahydrochloride to develop the peroxidase reaction. Counterstaining of the sections was carried out with hematoxylin, and they were then dehydrated in ethanol before mounting. Two independent investigators examined all tumor slides randomly. Five views were examined per slide, and 100 cells were observed per view at $\mathrm{x} 400$ magnification. Immunostaining of Mig- 6 was scored following a semi-quantitative scale by evaluating in representative tumor areas. The intensity of Mig- 6 staining was scored as 0 (no staining); 1 (weak) and 2 (marked). Percentage scores were assigned as 1, 1-25\%; 2, 26-50\%; and 3, 51-100\%. The scores of each tumor sample were multiplied to give a final score of $0-6$ and the total expression of Mig- 6 was determined as either negative or low expression (-), score $<3$ or positive expression or high expression (+), score $\geq 3$.

Western blot analysis. Total protein from cell lines were extracted in lysis buffer (Thermo Fisher Scientific) and quantified using the Bradford method. Sixty micrograms of protein were separated by SDS-PAGE (12\%). After transferring, the polyvinylidene fluoride (PVDF) membranes (Millipore, Billerica, MA, USA) were incubated overnight at $4^{\circ} \mathrm{C}$ with the following antibodies: Mig-6 (1:2,000; Sigma), Bcl-2 and $\beta$-actin (1:500; Santa Cruz Biotechnology, Santa Cruz, CA, USA), anti-phospho-ERK (1:2,000; Cell Signaling Technology, Danvers, MA, USA). After incubation with peroxidase-coupled anti-mouse or rabbit IgG (Santa Cruz Biotechnology) at $37^{\circ} \mathrm{C}$ for $2 \mathrm{~h}$, bound proteins were visualized using ECL (Thermo Fisher Scientific) and detected using BioImaging Systems (UVP Inc., Upland, CA, USA). The relative protein levels were calculated based on $\beta$-actin as the loading control.

Quantitative real-time PCR (SYBR-Green method). Quantitative real-time PCR was performed using SYBR-Green PCR master mix in a total volume of $20 \mu 1$ on 7900HT Fast Real-Time PCR System (both from Applied Biosystems) as follows: $95^{\circ} \mathrm{C}$ for $30 \mathrm{sec}, 40$ cycles of $95^{\circ} \mathrm{C}$ for $5 \mathrm{sec}, 60^{\circ} \mathrm{C}$ for $30 \mathrm{sec}$. A dissociation step was performed to generate a melting curve to confirm the specificity of the amplification. $\beta$-actin was used as the reference gene. The relative levels of gene expression were represented as $\Delta \mathrm{Ct}=\mathrm{Ct}$ gene - $\mathrm{Ct}$ reference, and the fold-change of gene expression was calculated by the $2^{-\Delta \Delta \mathrm{Ct}}$ method.

Cell apoptosis experiments. Apoptosis was detected using an Annexin V-FITC/PI double staining kit (KeyGen, Nanjing, China). Cells were harvested and washed twice with cold phosphate-buffered saline (PBS) by gentle shaking. Cells were then resuspended and added to binding buffer $(1 \mathrm{X})$; cell density was adjusted to $2-5 \times 10^{5} / \mathrm{ml}$. In the dark, $5 \mu 1$ Annexin V-FITC 

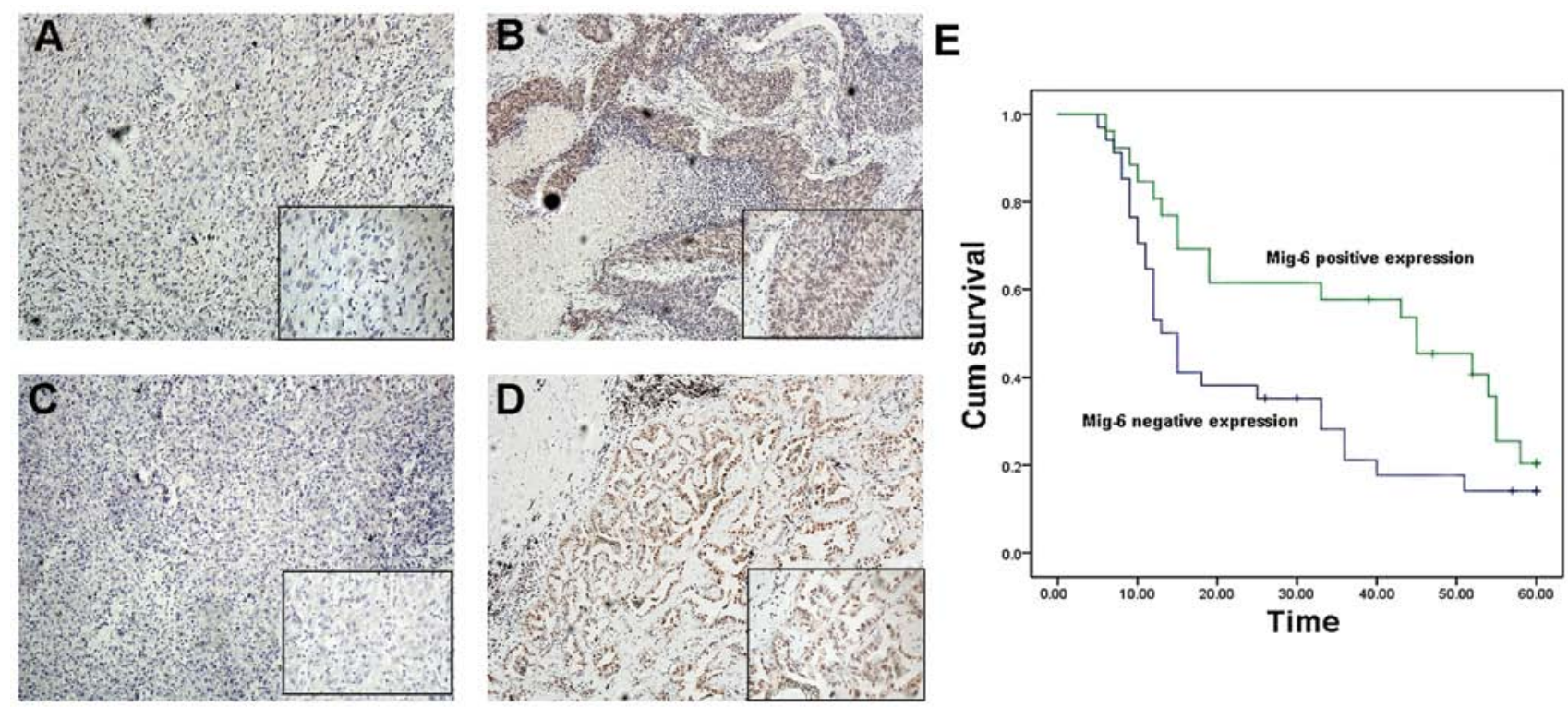

Figure 1. Expression of Mig-6 in NSCLCs and prediction of poor prognosis. Immunohistochemistry was performed to determine the expression of Mig-6. (A) Weak Mig-6 immunostaining in squamous cell carcinoma. (B) Strong Mig-6 immunostaining in squamous cell carcinoma. (C) Weak Mig-6 expression in adenocarcinoma. (D) Strong Mig-6 expression in adenocarcinoma. (E) Disease-free survival after surgery was compared between Mig-6-positive (n=26) and Mig-6-negative ( $\mathrm{n}=34)$ NSCLC patients. Mig-6, mitogen-inducible gene-6.

was added to the cell suspension volume of $195 \mu \mathrm{l}$ and incubated for $10 \mathrm{~min}$ at room temperature before the addition of $190 \mu \mathrm{l}$ binding buffer (1X) and $10 \mu \mathrm{l}$ PI. Ten thousand events per sample were acquired using a FACScan flow cytometer and the percentage of cell apoptosis was analyzed using CellQuest analysis software (both from Becton-Dickinson, San Jose, CA, USA).

Cell proliferation test. Cell proliferation assay was performed using Cell Counting Kit- $8^{\circledR}$ solution (Dojindo, Gaithersburg, MD, USA) according to the manufacturer's protocol. Briefly, cells were seeded at a concentration of $5 \times 10^{3}$ cells $/ 100 \mu \mathrm{l} /$ well in 96 -well culture plates and treated with $10 \mu \mathrm{l} /$ well of Cell Counting Kit- $8^{\circledR}$ solution during the last $4 \mathrm{~h}$ of the culture. Optical density of the wells was measured at $450 \mathrm{~nm}$ using a microplate reader.

Colony formation assay. For colony formation assays, cells were grown on $6 \mathrm{~cm}$ dishes at a density of 5,000 cells/dish, and transfected with PC or Mig-6. Colonies were washed twice with PBS, fixed, and stained with formaldehyde-crystal violet 13-15 days after transfection. All experiments were independently repeated a minimum of three times under identical conditions.

Matrigel invasion assay. Cell invasion assay was performed using a 24-well Transwell chamber with a pore size of $8 \mu \mathrm{m}$ (Costar, Cambridge, MA, USA). The inserts were coated with $20 \mu 1$ Matrigel (1:3 dilution; BD Biosciences, San Jose, CA, USA). Forty-eight hours after the transfection, cells were trypsinized and $3 \times 10^{5}$ cells in $100 \mu \mathrm{l}$ of serum-free medium were transferred to the upper Matrigel chamber and incubated for $16 \mathrm{~h}$. After incubation, the non-invaded cells on the upper membrane surface were removed with a cotton tip, and the cells that passed through the filter were fixed with
4\% paraformaldehyde and stained with hematoxylin. The number of invaded cells was counted in 10 randomly selected high power fields under the microscope. This experiment was performed in triplicate.

Statistical analysis. SPSS version 19.0 for Windows was used for all analyses. The $\chi^{2}$ test was used to examine possible correlations between Mig-6 expression and clinicopathological factors. The Kaplan-Meier method was used to estimate the probability of patient survival, and differences in the survival of subgroup of patients were compared by using log-rank test. The Cox regression mode laws were used for multivariate analysis. Student's t-test was used to compare differences between groups. p-values were based on the two-sided statistical analysis, and $\mathrm{p}<0.05$ was considered to indicate a statistically significant difference.

\section{Results}

Correlation between Mig-6 expression and survival. We first stratified these 150 lung cancer patients as Mig-6-positive and -negative groups according to the Mig-6 staining of tumor sections (Fig. 1A-D). As shown in Table I, statistical differences were found between Mig-6 expression and the characteristics of tumor size $(\mathrm{T} 1+\mathrm{T} 2 \mathrm{vs}$. T3+T4, $\mathrm{p}=0.002)$, differentiation (high vs. poor-moderate, $\mathrm{p}=0.002)$, TNM stage (I+II vs. III+IV, $\mathrm{p}=0.014)$, as well as histological type (adenocarcinoma vs. squamous cell carcinoma, $\mathrm{p}=0.005$ ). We followed up on 60 patients and divided them into two groups according to Mig-6 expression. Patients with high expression of Mig-6 had a statistically significantly longer survival (median survival, 45 \pm 11.132 months; 95\% CI, 23.18266.818 months) than those with low expression of Mig- 6 (median survival, $13 \pm 1.458$ months; 95\% CI, 10.143-15.857 months). Furthermore, a multivariate analysis using a Cox regression model indicated that pTNM stage and loss of Mig- 6 expression 
Table I. Relationship between Mig-6 expression in NSCLC and clinical pathological factors.

\begin{tabular}{|c|c|c|c|c|}
\hline \multirow[b]{2}{*}{ Characteristics } & \multirow[b]{2}{*}{ Patients } & \multicolumn{3}{|c|}{ Mig-6 } \\
\hline & & $\begin{array}{c}\text { Positive } \\
\text { n (\%) }\end{array}$ & $\begin{array}{c}\text { Negative } \\
\text { n (\%) }\end{array}$ & P-value \\
\hline Age (years) & & & & 1.000 \\
\hline$<60$ & 80 & $37(46.25)$ & $43(53.75)$ & \\
\hline$\geq 60$ & 70 & $33(47.14)$ & $37(52.86)$ & \\
\hline Gender & & & & 0.324 \\
\hline Male & 88 & 38 (43.18) & $50(56.82)$ & \\
\hline Female & 62 & $32(51.61)$ & $30(48.39)$ & \\
\hline Histology & & & & 0.005 \\
\hline $\mathrm{ADC}$ & 81 & $52(64.20)$ & $29(35.80)$ & \\
\hline $\mathrm{SCC}$ & 69 & $28(40.58)$ & $41(59.42)$ & \\
\hline Differentiation & & & & 0.002 \\
\hline Well & 47 & $31(65.96)$ & $16(34.04)$ & \\
\hline Moderate-Poor & 103 & $39(37.86)$ & $64(62.14)$ & \\
\hline TNM stage & & & & 0.014 \\
\hline I+II & 80 & $45(56.25)$ & $35(43.75)$ & \\
\hline III+IV & 70 & $25(35.71)$ & 45 (64.29) & \\
\hline Tumor size & & & & 0.002 \\
\hline $\mathrm{T} 1+\mathrm{T} 2$ & 65 & $40(60.71)$ & $25(39.29)$ & \\
\hline $\mathrm{T} 3+\mathrm{T} 4$ & 85 & $30(42.86)$ & $55(57.14)$ & \\
\hline Nodal status & & & & 0.323 \\
\hline - & 90 & $39(43.33)$ & $51(56.67)$ & \\
\hline+ & 60 & $31(51.67)$ & $29(48.33)$ & \\
\hline EGFR status & & & & 0.000 \\
\hline Negative & 62 & $41(66.67)$ & $21(33.33)$ & \\
\hline Positive & 88 & $29(34.62)$ & $59(65.38)$ & \\
\hline
\end{tabular}

Table II. Multivariate analysis for predictive factors in patients with NSCLC (Cox regression model).

\begin{tabular}{lrccc}
\hline Factors & & \multicolumn{3}{c}{$95 \%$ CI } \\
& Wald & $\operatorname{Exp}(\mathrm{B})$ & for $\exp (\mathrm{B})$ & P-value \\
\hline Tissue histology & 3.655 & 1.986 & $0.983-4.012$ & 0.056 \\
Differentiation & 0.209 & 0.839 & $0.395-1.781$ & 0.648 \\
Gender & 0.009 & 1.030 & $0.554-1.915$ & 0.925 \\
Age (years) & 2.644 & 1.028 & $0.994-1.064$ & 0.104 \\
TNM stage & 25.251 & 3.715 & $2.227-6.198$ & 0.000 \\
Mig-6 expression & 7.927 & 0.329 & $0.152-0.713$ & 0.005 \\
\hline
\end{tabular}

were independent, unfavorable prognostic factors [TNM stage: $\operatorname{Exp}(\mathrm{B}), 3.715,95.0 \%$ CI, 2.227-6.198, p=0.000; Mig-6 expression: $\operatorname{Exp}(\mathrm{B}), 0.329,95.0 \% \mathrm{CI}, 0.152-0.713, \mathrm{p}=0.005)$ (Table II)].

Mig- 6 promotes apoptosis of NSCLC cell lines by inhibiting the expression of Bcl-2. To determine whether Mig-6 influences

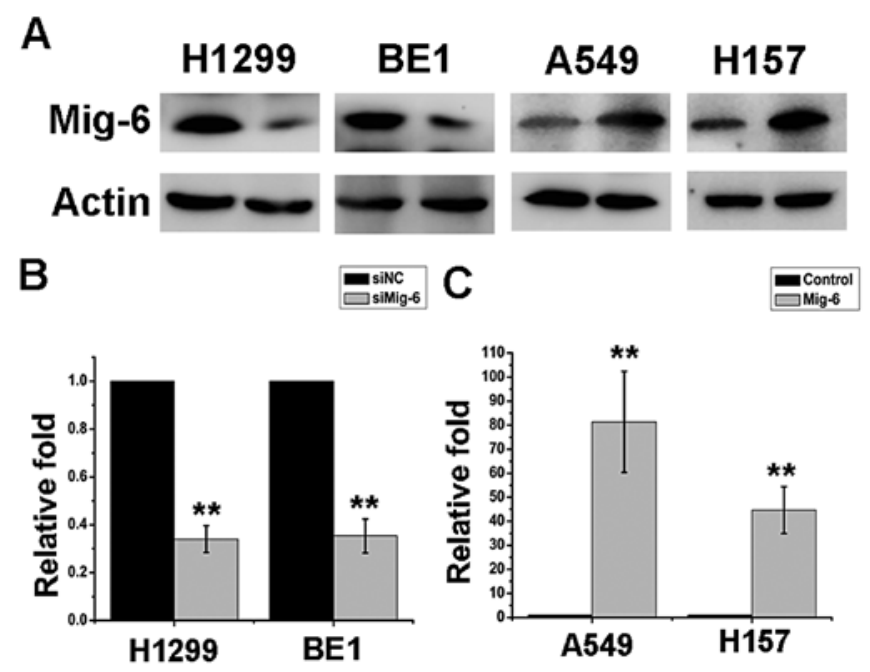

Figure 2. Mig-6 depletion and overexpression in lung cancer cells (A) Western blot analysis of knockdown efficiency of Mig-6 siRNA in the H1299 and BE1 cell lines and Mig- 6 transfected efficiency in A549 and H157 cell lines. (B) Knockdown efficiency as assessed by real-time PCR $48 \mathrm{~h}$ after transfection in H1299 and BE1 cell lines. (C) Transfected efficiency as assessed by real-time PCR $48 \mathrm{~h}$ after transfection in A549 and H157 cell lines. Mig-6, mitogen-inducible gene-6.

the apoptosis of NSCLC cells, we explored Mig-6 knockdown and overexpression. Interference efficiency was examined by mRNA and protein expression levels after $48 \mathrm{~h}$ of transfection treatment (Fig. 2A-C). Annexin V staining was performed using flow cytometry to determine the effect of Mig- 6 on apoptosis in NSCLC cells. Mig- 6 knockdown decreased apoptosis in H1299 and BE1 cells (H1299 control vs. Mig-6 siRNA: $11.54 \pm 0.65$ vs. $6.10 \pm 1.29 \%, \mathrm{p}<0.01$; BE1 control vs. Mig-6 siRNA: $12.63 \pm 0.96$ vs. $6.42 \pm 0.83 \%$, p<0.01) (Fig. 3A). Mig-6 overexpression increased apoptosis in A549 and H157 cells (A549 empty vector vs. Mig-6 plasmid: 7.05 \pm 0.82 vs. $13.98 \pm 0.79 \%, \mathrm{p}<0.01$; H157 empty vector vs. Mig-6 plasmid: $12.46 \pm 0.87$ vs. $17.35 \pm 1.44 \%, \mathrm{p}<0.05$ ) (Fig. 3B).

It has been established that apoptosis within cells is chiefly involved in the expression of Bcl-2, Bax, or p53 (15-17). To determine a possible mechanism by which Mig- 6 promotes apoptosis of NSCLC cells, western blotting was performed. As shown in Fig. $3 \mathrm{C}$ the expression at both the protein and mRNA levels of anti-apoptotic Bcl-2 was upregulated in the Mig-6 depletion group but downregulated in the Mig-6 overexpression group. There were no significant differences in Bax and p53 expression between each group (data not shown). These results indicated that the function of Mig- 6 as a promotor of apoptosis in NSCLC cells is predominantly implemented possibly by the pathways of Bcl-2 but not Bax and p53.

Mig-6 downregulates Bcl-2 expression through the ERK signaling pathway. Our previous study confirmed that the suppression of Mig- 6 by a specific siRNA led to a marked increase in EGFR-ERK signaling (14). Numerous reports have shown that the ERK pathway plays an important role in the apoptosis of NSCLC (18-20). We investigated whether ERK mediated Mig- 6 induction of Bcl-2 downregulation. Cells were treated with PD98059, a selective inhibitor of MEK that disrupts the activation of downstream ERK, for $1 \mathrm{~h}$. We found 
A

siNC siMig-6

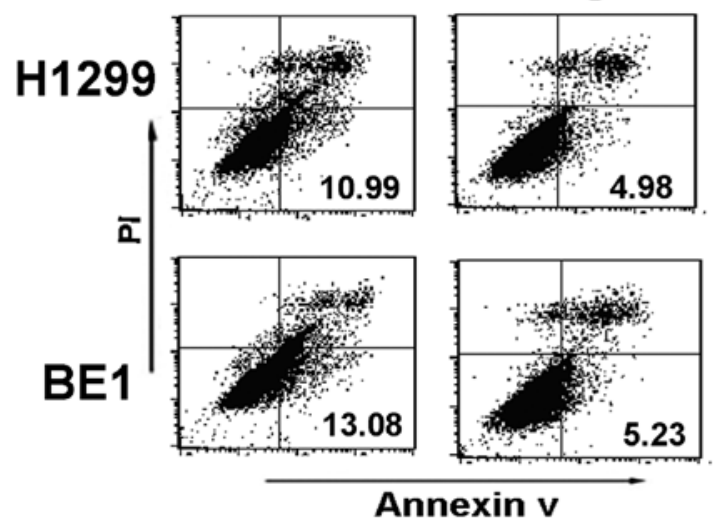

H1299

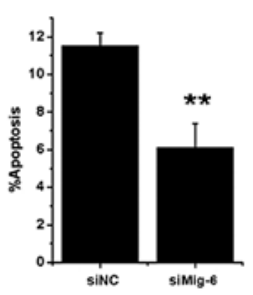

\section{BE1}

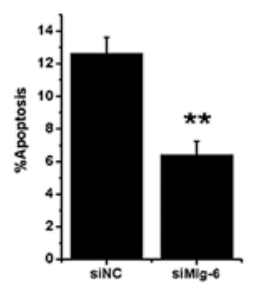

B

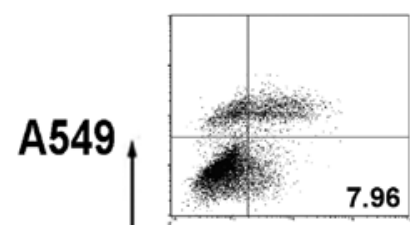

PC

$\bar{a}$
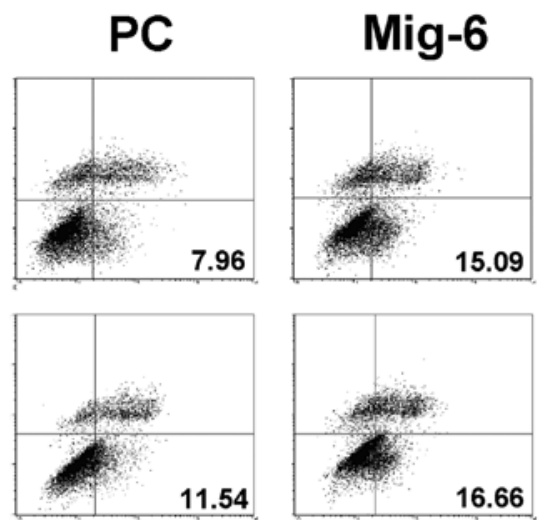

A549

H157

H157
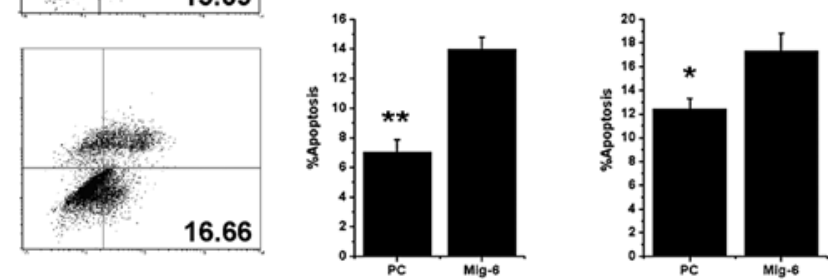

Annexin v
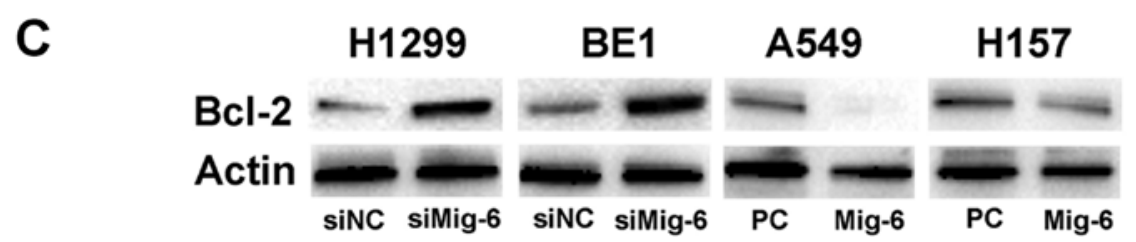

Figure 3. Effect of Mig-6 on apoptosis in lung cancer cells. (A) H1299 and BE1 were transfected with negative control or Mig-6 siRNA for $48 \mathrm{~h}$. Cells were harvested and then subjected to apoptosis experiments using Annexin V staining. The percentage of apoptotic cells is expressed as the means \pm SD of three independent experiments. (B) A549 and H157 cells were transfected with pcDNA3 vector-, or pcDNA3-Mig-6 overexpression vector-, for $48 \mathrm{~h}$. The percentage of apoptotic cells is expressed as the means \pm SD of three independent experiments. (C) Cells were transfected with siRNA targeted against Mig- 6 or pcDNA3Mig-6 overexpression vector, and cell lysates were analyzed by using western blotting for the presence of Bcl-2 proteins. Mig-6, mitogen-inducible gene-6.

that basal Bcl-2 production and anti-apoptosis ability were inhibited by treatment of H1299 and BE1 cells with $50 \mathrm{~mol} / \mathrm{l}$ PD98059. Furthermore, PD98059 significantly abolished Mig-6 siRNA-mediated Bcl-2 production and anti-apoptotic effects (Fig. 4A). Consequently, we overexpressed Mig-6 in A549 and H157 cells, we also blocked ERK activities using PD98059. As shown in Fig. 4B, Mig-6 and PD98059 can inhibit the expression of P-ERK and Bcl-2. Similar to Mig-6 overexpression, inhibition of ERK by PD98059 can also promote apoptosis of both cell lines. To further validate the role of ERK signaling in Mig-6-induced apoptosis, we used siRNAs to deplete ERK1/2 expression in H1299 and BE1 cells. Then, we transfected Mig-6 siRNA and examined Bcl-2 protein expression, as well as the apoptosis ability of cells. As shown in Fig. 4C, the effect of Mig-6 siRNA-mediated Bcl-2 production and anti-apoptotic effects were abolished in ERK1/2-depleted H1299 and BE1 cell lines.

Effect of Mig-6 upregulation on the proliferation and invasive potential of transfected cells. A549 and H157 cells were used in the present study to investigate the effects of Mig-6 upregulation on proliferation and invasion. These cells have low endogenous Mig-6 protein levels. The Mig-6 expression levels were unchanged upon transient transfection with the empty vector, whereas pcDNA3-Mig-6 plasmid significantly upregulated both mRNA as well as protein expression levels in 
A

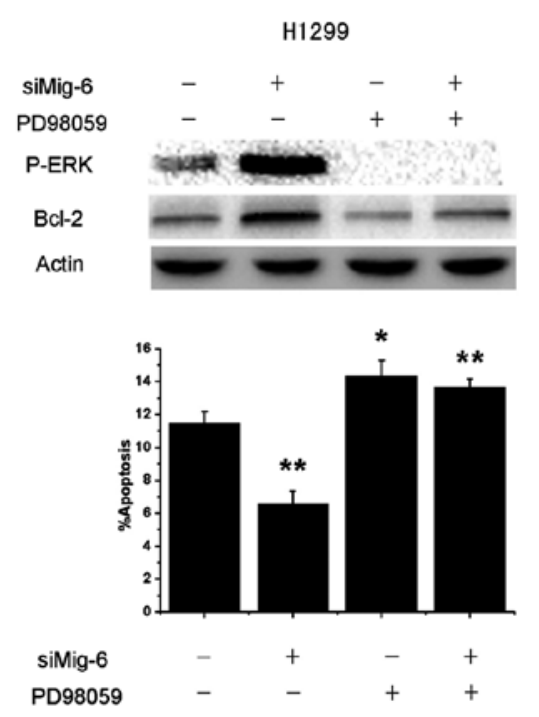

C
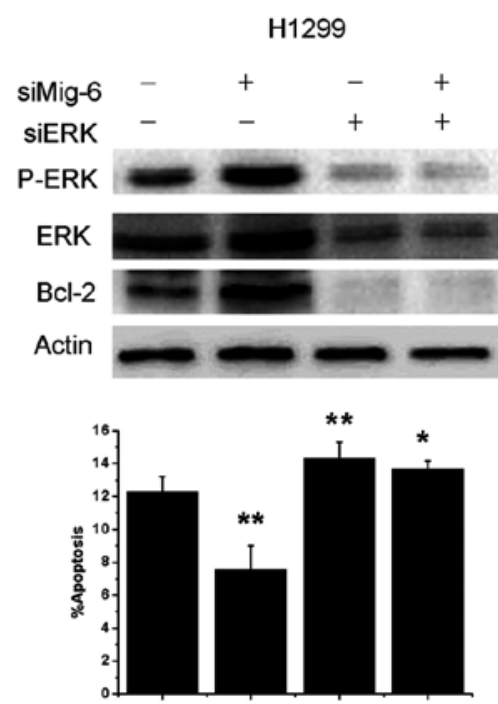

$\begin{array}{ccccc}\text { siMig-6 } & - & + & - & + \\ \text { siERK } & - & - & + & +\end{array}$
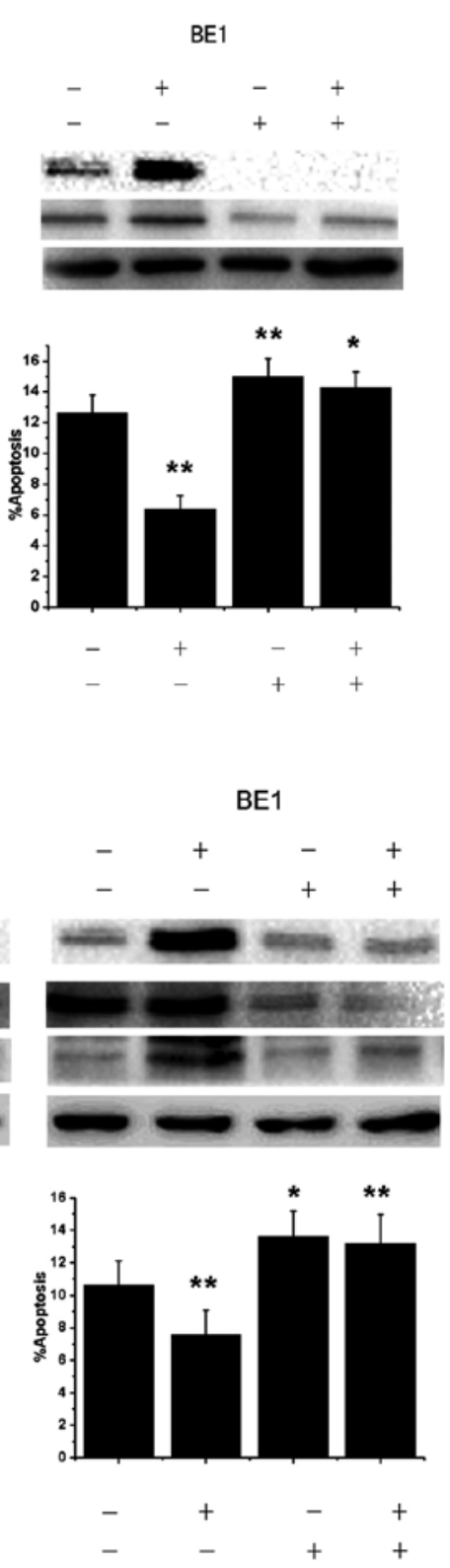

B
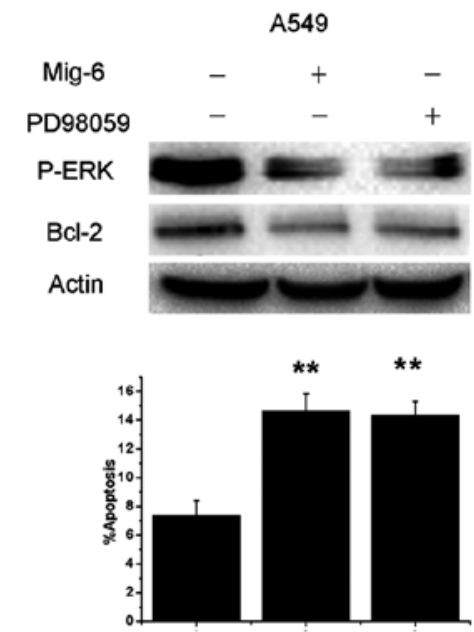

Mig-6

PD98059

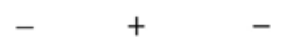

$+\quad+$

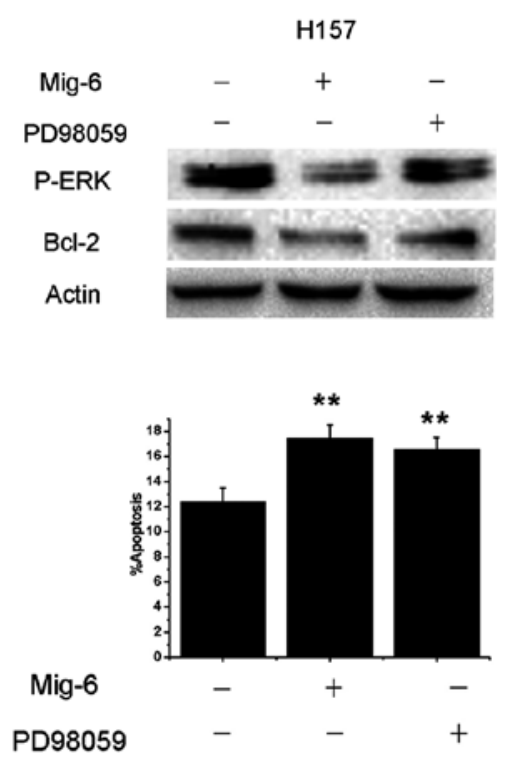

Figure 4. Mig-6 downregulates Bcl-2 expression and promotes cell apoptosis through the ERK signaling pathway. (A) H1299 and BE1 cells were pretreated with Mig-6 siRNA for $48 \mathrm{~h}$ and the MEK inhibitor, PD98059 (50 mol/l) for another $1 \mathrm{~h}$, and whole cell lysates were analyzed for the protein levels of p-ERK and Bcl-2. Cell apoptosis was evaluated using Annexin V staining. (B) A549 and H157 cells were transfected with either PC or Mig-6, followed by PD98059 treatment. Levels of p-ERK, Bcl-2 were determined. Cell apoptosis was evaluated using Annexin V staining. (C) Mig-6 siRNA was transfected in H1299 and BE1 cells or in ERK1/2-depleted H1299 and BE1 cells. Levels of p-ERK, ERK and Bcl-2 were determined by using western blot analysis. Cell apoptosis was evaluated using Annexin V staining. Each bar represents the means \pm SD of three independent experiments. ${ }^{*}$ p $<0.05$. Mig- 6 , mitogen-inducible gene-6.

the A549 and H157 cell lines (Fig. 2A). Next, we examined the cell growth rate. With pcDNA3-Mig-6 plasmid transfection, A549 cells displayed reduced growth rates when compared to the control (Fig. 5A). Similar to A549 cells, we found that upregulation of Mig- 6 also reduced the growth rate of H157 cells (Fig. 5A). We utilized an independent method, colony formation assay, to validate the antiproliferative effects of Mig-6 in lung cancer cells. pcDNA3-Mig-6 plasmid led to a clear reduction of the colony formation capacity of two tested lung cancer cell lines compared to empty vector control (empty vector vs. Mig-6 plasmid: A549, 210.33 \pm 19.66 vs. 84.33 \pm 10.97 , $\mathrm{p}<0.01$; H157, 163.33 \pm 13.01 vs. $119 \pm 17.35$, p<0.01) (Fig. 5B).
These overexpresssion studies demonstrated that Mig-6 inhibits tumor cell proliferation. To further examine whether Mig- 6 contributes to the invasive capabilities of NSCLC cells, we conducted Matrigel invasion assays. The expression of Mig-6 was enhanced in transfected cells in comparison to the control cells. Our results demonstrated that the invasive capabilities of Mig-6 overexpressed A549 and H157 cancer cells were reduced compared to the control cells (empty vector vs. Mig-6 plasmid: A549, 58 \pm 8 vs. $34.33 \pm 4.04$, p <0.05; H157, $53.67 \pm 5.13$ vs. $16.33 \pm 3.21, \mathrm{p}<0.01$ ) (Fig. 5C). Collectively, these results demonstrate that increased expression of Mig-6 significantly inhibited invasion of A549 and H157 cells. 
A
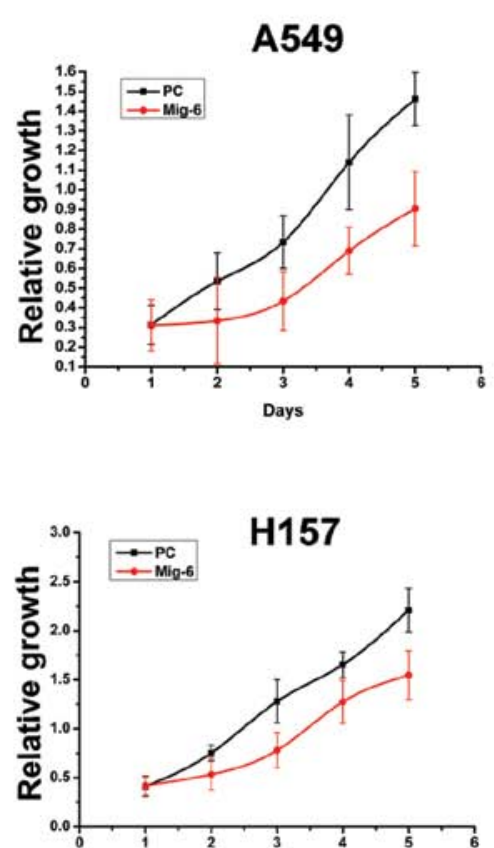

B

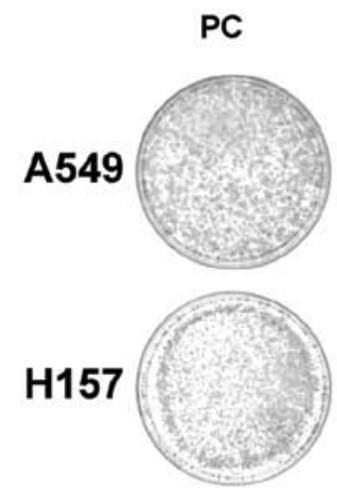

A549

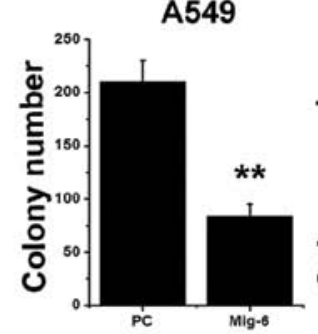

Mig-6

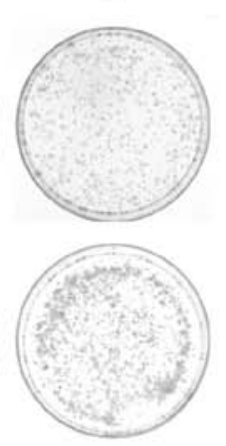

A549

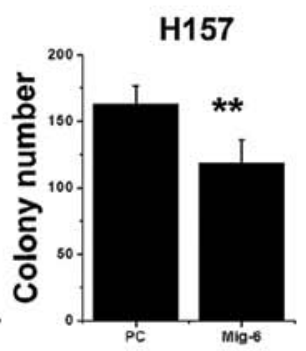

C
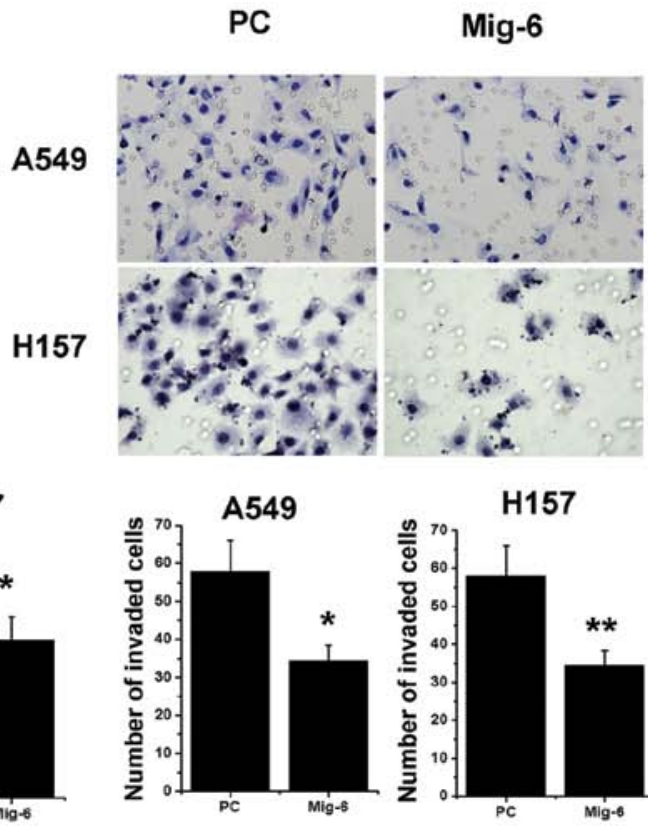

Figure 5. Overexpression of Mig-6 in A549 and H157 cell lines induces proliferation and inhibits invading ability. (A) Cell viability was determined by the CCK-8 assay, as described in Materials and methods. The data are means \pm SD of three independent experiments. (B) (Upper) A549 and H157 cells transduced with PC or Mig-6 were subjected to colony formation assay; (Lower) histogram quantification. Error bars indicate \pm SD $\left({ }^{*} \mathrm{p}<0.05,{ }^{* *} \mathrm{p}<0.01\right)$. (C) (Upper) PC or Mig-6 treatment inhibited measurable cell invasion in both cell lines (x400). (Lower) The bar graph indicates the mean cell number among the images, and the standard deviation was calculated from the average of cells in the 10 fields. ${ }^{* *} \mathrm{p}<0.01$. Mig- 6 , mitogen-inducible gene- 6 .

\section{Discussion}

Mig-6 downregulation has been observed in various types of cancer. Loss of Mig- 6 in mice leads to impaired differentiation of epidermal keratinocytes and these Mig- 6 ablation mice are highly susceptible to carcinogen-induced formation of papillomas and melanomas (6). Disruption of Mig-6 in mice also causes lung, gallbladder and bile duct carcinogenesis (7). In papillary thyroid cancer, tumor size was inversely correlated with Mig-6 expression (21). In immunohistochemical analysis using a tissue microarray of 111 liver cancer patients, Reschke et al found that Mig-6 expression was barely detectable in the majority of analyzed tumors (64\%) (22). We previously demonstrated that Mig- 6 expression was downregulated or absent in NSCLC (14).

Despite the strong association between Mig-6 expression and cancer, reports on Mig-6 expression-based outcome in tumor patients are limited. Using real-time polymerase chain reaction (PCR), Ruan et al found that Mig-6 expression is independently predictive of disease-free-survival in $\mathrm{BRAF}^{\mathrm{V} 600 \mathrm{E}}$ patients (23). Amatschek et al demonstrated that the Mig- 6 gene is downregulated relative to normal tissues in patients with short survival using cDNA microarray analysis (13). These data suggest that Mig-6 expression may correlate closely with poor prognosis in some types of human cancer.

However, the protein expression status of Mig- 6 correlation with the outcome of the cancer patient has not been elucidated. In the present study, we found Mig-6 downregulation correlated with a poor prognosis in patients. We also found Mig-6 expression was lower in NSCLCs of poor differentiation and advanced stage. Cox regression multivariate analysis showed tumor stage and Mig- 6 were the strongest predictors of survival. Our results suggest a critical effect of Mig-6 silencing in tumorigenesis and aggression of NSCLC.

The biological functions of Mig- 6 low expression have been investigated in several cell lines. Downregulation of Mig- 6 promotes proliferation and invasion in glioblastoma multiforme (GBM) and PTC cell lines $(21,24)$. Mig-6 is also an endogenous inhibitor of EGF-induced cell migration in human live cancer cell line HepG2 (22). Our previous study showed that Mig- 6 specific siRNA promotes proliferation and invasion of H1299 and BE1 cells. However, how restored Mig-6 expression in lung cancer cells affects proliferation and invasion of A549 and H157 cells has not been fully clarified. In the present study, we further examined the effects of exogenously transfected Mig- 6 on proliferation, invasion and apoptosis of A549 and H157 cells. We concluded that upregulated Mig-6 decreased proliferated and invasive cells, and increased apoptotic cells. Our data suggest that exogenously expressed Mig-6 may effectively inhibit progression of lung cancer.

The role of Mig-6 in apoptosis remains unclear. Our findings that Mig- 6 promotes apoptosis in NSCLC cell lines are in concordance with previous reports in endometrial cancer (25), but appear inconsistent with the findings reported in breast cancer cell lines (11). This observation led us to extend our investigation into the underlying mechanisms of Mig-6induced apoptosis. Bcl-2 was one of the regulators of the cell intrinsic apoptosis pathway, which regulates the integrity of the outer mitochondria membrane (26). In agreement with results from different cell models, our results showed that Mig-6 specific siRNA prevented apoptosis, resulting in an increase in the expression of anti-apoptotic Bcl-2. Next, we investigated 
how Mig-6 specific siRNA increases expression of Bcl-2. Our previous study showed that Mig-6 specific siRNA promoted P-ERK expression. P-ERK, as a downstream factor of Mig-6, is involved in the regulation of cell proliferation, differentiation, apoptosis and cell cycle arrest, as well as the induction of drug resistance $(27,28)$. The Mig-6 siRNA-mediated Bcl-2 overexpression and anti-apoptotic effects can be reversed by ERK inhibitor PD98059 or ERK siRNA. Furthermore, Mig-6 overexpression or use of PD98059 can all reduce the expression of P-ERK, inhibit the Bcl-2 production, which lead to the increase of the apoptosis of cells. These results demonstrate that the effect of Mig- 6 on apoptosis involved in the expression of Bcl-2 may occur via the ERK pathway in human NSCLC cells.

In conclusion, we have identified Mig- 6 as a potential biomarker for evaluation of tumor prognosis of lung cancer. Our findings also suggest the potential important role of Mig-6 in the control of lung cell apoptosis, an activity that may be responsible, at least in part, for the development and/or progression of lung cancer.

Meanwhile, the present study provides further evidence for our previous results that Mig- 6 plays a critical role in inhibiting lung cancer cell proliferation and invasion. The upregulation of Mig-6 may provide a helpful strategy for inhibitory therapies of NSCLC.

\section{Acknowledgements}

The authors thank Dr Oreste Segatto (Regina Elena Cancer Institute, Via Delle Messi d'Oro 156, Rome 00158, Italy) for kindly providing the pcDNA3 vector- and pcDNA3-Mig-6 overexpression vector. This study was supported by grants from the National Natural Science Foundation of China (no. 30972967), and the Specialized Research Fund for the Doctoral Program of Higher Education (no. 20092104110018), and the Program for Liaoning Excellent Talents in University. We thank International Science Editing (Shannon Free ZoneWest, Shannon, Co., Clare Ireland) for the critical review of the manuscript.

\section{References}

1. Zhang YW, Vande Woude GF: Mig-6, signal transduction, stress response and cancer. Cell Cycle 6: 507-513, 2007.

2. Ogunbiyi OA, Goodfellow PJ, Gagliardi G, et al: Prognostic value of chromosome 1p allelic loss in colon cancer. Gastroenterology 113: 761-766, 1997.

3. Koshikawa K, Nomoto S, Yamashita K, Ishigure K, Takeda S and Nakao A: Allelic imbalance at 1p36 in the pathogenesis of human hepatocellular carcinoma. Hepatogastroenterology 51: 186-191, 2004.

4. Tseng RC, Chang JW, Hsien FJ, et al: Genomewide loss of heterozygosity and its clinical associations in non small cell lung cancer. Int J Cancer 117: 241-247, 2005.

5. Jeong JW, Lee HS, Lee KY, et al: Mig-6 modulates uterine steroid hormone responsiveness and exhibits altered expression in endometrial disease. Proc Natl Acad Sci USA 106: 8677-8682, 2009.

6. Ferby I, Reschke M, Kudlacek O, et al: Mig6 is a negative regulator of EGF receptor-mediated skin morphogenesis and tumor formation. Nat Med 12: 568-573, 2006.
7. Zhang YW, Staal B, Su Y, et al: Evidence that $M I G-6$ is a tumorsuppressor gene. Oncogene 26: 269-276, 2007.

8. Jin N, Gilbert JL, Broaddus RR, Demayo FJ and Jeong JW: Generation of a Mig- 6 conditional null allele. Genesis 45: 716-721, 2007.

9. Anastasi S, Sala G, Huiping C, et al: Loss of RALT/MIG-6 expression in ERBB2-amplified breast carcinomas enhances ErbB-2 oncogenic potency and favors resistance to Herceptin. Oncogene 24: 4540-4548, 2005.

10. Anastasi S, Fiorentino L, Fiorini M, et al: Feedback inhibition by RALT controls signal output by the ErbB network. Oncogene 22: 4221-4234, 2003.

11. Xu D, Makkinje A and Kyriakis JM: Gene 33 is an endogenous inhibitor of epidermal growth factor (EGF) receptor signaling and mediates dexamethasone-induced suppression of EGF function. J Biol Chem 280: 2924-2933, 2005.

12. Anastasi S, Baietti MF, Frosi Y, Alema S and Segatto O: The evolutionarily conserved EBR module of RALT/MIG6 mediates suppression of the EGFR catalytic activity. Oncogene 26: 7833-7846, 2007.

13. Amatschek S, Koenig U, Auer H, et al: Tissue-wide expression profiling using cDNA subtraction and microarrays to identify tumor-specific genes. Cancer Res 64: 844-856, 2004.

14. Li Z, Dong Q, Wang Y, Qu L, Qiu X and Wang E: Downregulation of Mig-6 in nonsmall-cell lung cancer is associated with EGFR signaling. Mol Carcinog 51: 522-534, 2012.

15. Wu S, Xing W, Peng J, et al: Tumor transfected with CCL21 enhanced reactivity and apoptosis resistance of human monocyte-derived dendritic cells. Immunobiology 213: 417-426, 2008.

16. Ling YH, Liebes L, Jiang JD, et al: Mechanisms of proteasome inhibitor PS-341-induced $\mathrm{G}_{2}$-M-phase arrest and apoptosis in human non-small cell lung cancer cell lines. Clin Cancer Res 9: 1145-1154, 2003.

17. Kim JW, Ferris RL and Whiteside TL: Chemokine C receptor 7 expression and protection of circulating $\mathrm{CD} 8^{+} \mathrm{T}$ lymphocytes from apoptosis. Clin Cancer Res 11: 7901-7910, 2005.

18. Faber AC, Li D, Song Y, et al: Differential induction of apoptosis in HER 2 and EGFR addicted cancers following PI3K inhibition. Proc Natl Acad Sci USA 106: 19503-19508, 2009.

19. Liu Y, Yang Y, Ye YC, et al: Activation of ERK-p53 and ERK-mediated phosphorylation of Bcl-2 are involved in autophagic cell death induced by the c-Met inhibitor SU11274 in human lung cancer A549 cells. J Pharmacol Sci 118: 423-432, 2012.

20. Okamoto K, Okamoto I, Okamoto W, et al: Role of survivin in EGFR inhibitor-induced apoptosis in non-small cell lung cancers positive for EGFR mutations. Cancer Res 70: 10402-10410, 2010.

21. Lin CI, Du J, Shen WT, et al: Mitogen-inducible gene-6 is a multifunctional adaptor protein with tumor suppressor-like activity in papillary thyroid cancer. J Clin Endocrinol Metab 96: E554-E565, 2011.

22. Reschke M, Ferby I, Stepniak E, et al: Mitogen-inducible gene-6 is a negative regulator of epidermal growth factor receptor signaling in hepatocytes and human hepatocellular carcinoma. Hepatology 51: 1383-1390, 2010.

23. Ruan DT, Warren RS, Moalem J, et al: Mitogen-inducible gene-6 expression correlates with survival and is an independent predictor of recurrence in $\mathrm{BRAF}^{\mathrm{V} 600 \mathrm{E}}$ positive papillary thyroid cancers. Surgery 144: 908-914, 2008.

24. Ying H, Zheng H, Scott K, et al: Mig- 6 controls EGFR trafficking and suppresses gliomagenesis. Proc Natl Acad Sci USA 107: 6912-6917, 2010.

25. Kim TH, Franco HL, Jung SY, et al: The synergistic effect of Mig- 6 and Pten ablation on endometrial cancer development and progression. Oncogene 29: 3770-3780, 2010.

26. Ewings KE, Wiggins CM and Cook SJ: Bim and the pro-survival Bcl-2 proteins: opposites attract, ERK repels. Cell Cycle 6: 2236-2240, 2007.

27. Chen YR, Wang X, Templeton D, Davis RJ and Tan TH: The role of c-Jun N-terminal kinase (JNK) in apoptosis induced by ultraviolet $\mathrm{C}$ and $\gamma$ radiation. Duration of JNK activation may determine cell death and proliferation. J Biol Chem 271: 31929-31936, 1996.

28. Xia Z, Dickens M, Raingeaud J, Davis RJ and Greenberg ME: Opposing effects of ERK and JNK-p38 MAP kinases on apoptosis. Science 270: 1326-1331, 1995. 